\title{
Note: A simple sample transfer alignment for ultra-high vacuum systems
}

A. Tamtögl, E. A. Carter, D. J. Ward, N. Avidor, P. R. Kole, A. P. Jardine, and W. Allison

Citation: Review of Scientific Instruments 87, 066108 (2016); doi: 10.1063/1.4954728

View online: http://dx.doi.org/10.1063/1.4954728

View Table of Contents: http://aip.scitation.org/toc/rsi/87/6

Published by the American Institute of Physics

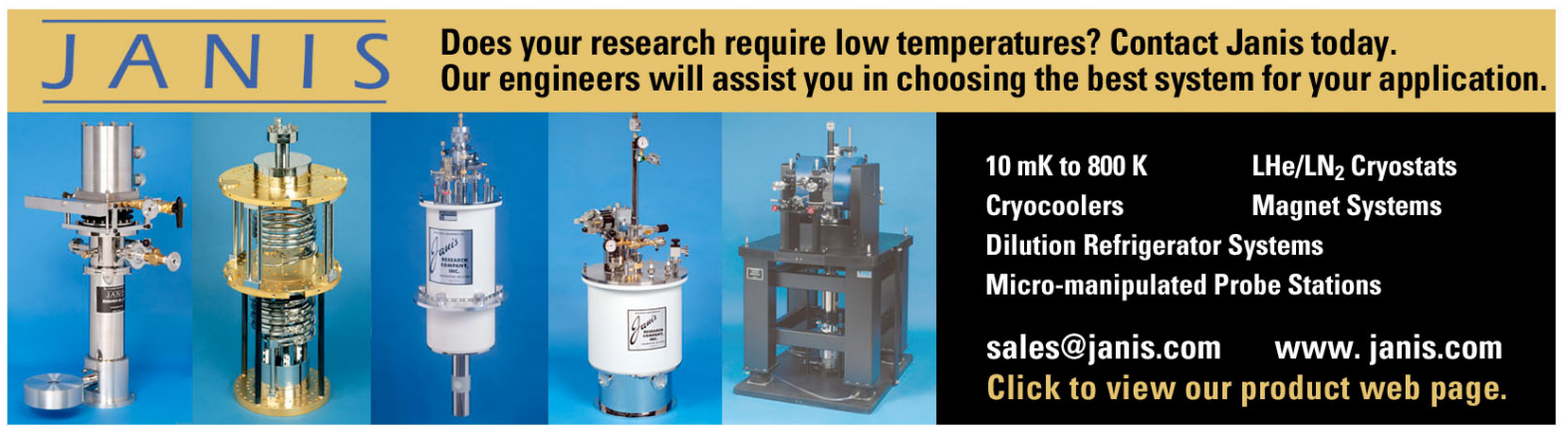




\title{
Note: A simple sample transfer alignment for ultra-high vacuum systems
}

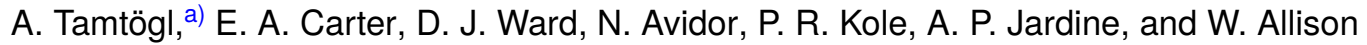 \\ Cavendish Laboratory, J. J. Thompson Avenue, Cambridge CB3 OHE, United Kingdom
}

(Received 23 April 2016; accepted 11 June 2016; published online 23 June 2016)

\begin{abstract}
The alignment of ultra-high-vacuum sample transfer systems can be problematic when there is no direct line of sight to assist the user. We present the design of a simple and cheap system which greatly simplifies the alignment of sample transfer devices. Our method is based on the adaptation of a commercial digital camera which provides live views from within the vacuum chamber. The images of the camera are further processed using an image recognition and processing code which determines any misalignments and reports them to the user. Installation has proven to be extremely useful in order to align the sample with respect to the transfer mechanism. Furthermore, the alignment software can be easily adapted for other systems. Published by AIP Publishing. [http://dx.doi.org/10.1063/1.4954728]
\end{abstract}

In order to study surface phenomena, scientists rely on well-defined systems which have been prepared under ultrahigh vacuum (UHV) conditions. Therefore, the sample which is to be examined is usually mounted on a manipulator inside a UHV chamber. To avoid breaking the vacuum in the apparatus and the need for subsequent baking of the chamber, a sample transfer mechanism is often used to introduce the sample into the chamber. Hence, the operational time of the apparatus is increased and the system can be easily switched between different samples. Reliable sample exchanges require correct alignment of the sample manipulator and the transfer tool. Users often find the process difficult, especially when direct observation of the sample is not possible.

The sample transfer system presented in this work is part of the Cambridge helium-3 spin-echo spectrometer: The apparatus combines the surface sensitivity of helium atom scattering $^{1,2}$ with the spin-echo technique, hence allowing examination of the surface dynamics of materials with atomic resolution, over time scales down to sub-picoseconds. ${ }^{3}$ The sample is mounted onto a custom made manipulator, which allows heating to $1400 \mathrm{~K}$ and cooling down to $100 \mathrm{~K}$ (using liquid nitrogen) or $40 \mathrm{~K}$ (using liquid helium). ${ }^{4}$ Because the spinecho technique relies on magnetic fields that are precisely defined, stray magnetic fields have to be brought to a minimum. External magnetic fields are shielded using nested mumetal housings. As a result, only one small window gives views into the main chamber, with no line-of-sight for direct observation during the sample transfer.

The manipulator provides alignment of the sample in the apparatus through translation as well as rotation. Linear translations along three axes $(x, y, z)$ and rotations around three axes $(\alpha, \beta, \gamma)$ are possible (see Fig. 1). The sample itself is mounted on a cartridge, which also contains electrical connections and facilities to heat and cool the sample. Both the sample and cartridge are exchanged in a sample transfer operation. The cartridge is fixed to the manipulator by a locking screw, giving good contact to a cooling reservoir, which improves

$\overline{\text { a)Electronic mail: tamtoeg1@gmail.com }}$ the thermal characteristics. The mechanical complexity of the manipulator and the need for close thermal contact generate technical challenges beyond those in simpler transfer systems.

The transfer system consists of two magnetically coupled linear/rotary drives (UHV Design, PP-series). One is used to operate the pickup tool and the second locks the cartridge to the manipulator (rear tool in Fig. 1). The pickup tool operates from
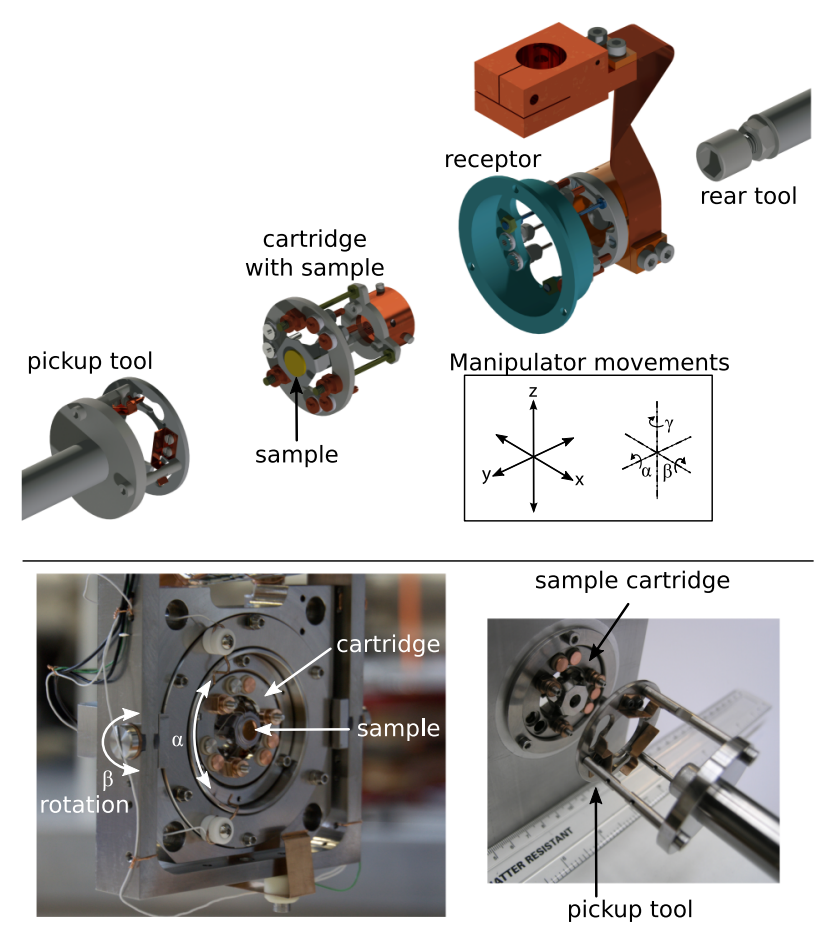

FIG. 1. Upper panel: Drawing of the sample transfer system. The sample which is mounted onto a cartridge is first attached to the pickup tool. The pickup tool is used to move the cartridge into the receptor on the manipulator. Then a hex tool (rear tool) on the rear side of the manipulator is used to tighten a locking screw which holds the cartridge in place. Finally the pickup tool can be detached and retracted. In order to insert the cartridge into the receptor it is crucial that the manipulator, which can be moved around six axes (linear translation along $(x, y, z)$ and rotation around $(\alpha, \beta, \gamma))$ is exactly aligned. Lower panel: Photo of the manipulator on the left and photo of the pickup tool facing the sample cartridge on the right. 
a separate load-lock chamber, connected to the main chamber by a gate valve. Transferring a sample to the manipulator occurs as follows: First, the cartridge and sample are attached to a pickup tool (see Fig. 1). The transfer chamber is then sealed and pumped down to $10^{-8}$ mbar, before opening the gate valve to the main chamber. The pickup tool is manipulated using the first linear/rotary drive to insert it into a bayonet-style receptor on the manipulator. Next, the hex-head on the rear tool is used to tighten the locking screw, fixing the cartridge in place. Finally, the pickup tool is detached and retracted so that the gate valve to the transfer chamber can be closed.

Insertion of the cartridge into the bayonet receptor on the manipulator is the critical step. It is essential that the manipulator and the pickup tool are aligned precisely during the operation. However, precise alignment is difficult without direct visual assistance. We overcome the difficulty in two stages: first the manipulator and pickup tool are aligned optically using a digital camera attached to the pickup tool; second, we replace the camera with the sample to be transferred. The camera is a simple USB webcam (Logitech, $640 \times 480$ pixels), which was stripped down (the housing was removed) and mounted on a dummy sample cartridge.

The mounting arrangement for the camera is shown in Fig. 2(a). Three white LEDs (round lens with $3 \mathrm{~mm}$ diameter, $2.9 \mathrm{~V}$ forward voltage, $10 \mathrm{~mA}$; in series with a $210 \Omega$ resistor) provide illumination. They were connected to the USB power terminals on the PCB of the webcam and fixed onto the mount (see Fig. 2(a)). Outgassing from trapped volumes inside the webcam is minimised by drilling a small hole in the camera lens casing and filing down one side of the mounting thread. A standard electrical feedthrough was used to transmit signals from inside the chamber to an external USB port. Our camera assembly is not designed to be compatible with UHV and is much simpler than other designs. ${ }^{5}$ When the camera is inserted into the load-lock chamber, it reaches a pressure of $2 \cdot 10^{-8}$ mbar (the pumping speed being $651 \mathrm{~s}^{-1}$ ). During operation the pressure increases slightly, but heat transfer from the electronic components of the webcam did not pose any problems even for an operation of up to an hour. Upon inserting the camera into the UHV chamber, the pressure rose to $1 \cdot 10^{-9}$ mbar. After the alignment procedure has been

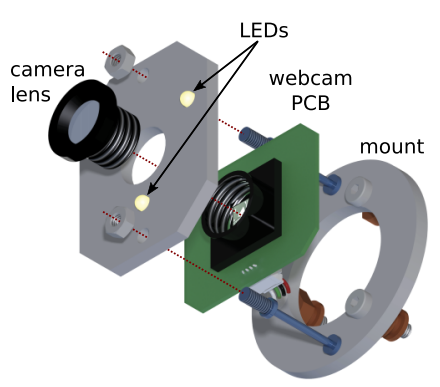

(a)

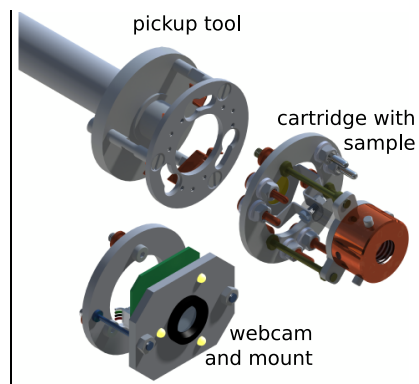

(b)
FIG. 2. Drawings of the camera and its mount which can be easily exchanged with the sample cartridge. (a) A simple webcam has been stripped down and fixed onto a mount similar to the sample cartridge. Three white LEDs have been added to provide lighting inside the chamber. (b) Both the camera mount and the sample cartridge are attached in the same way onto the pickup tool. Therefore it is very easy to switch back and forth between both options.

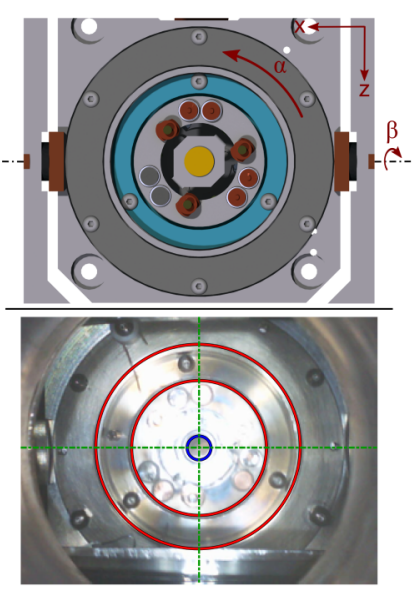

(a)

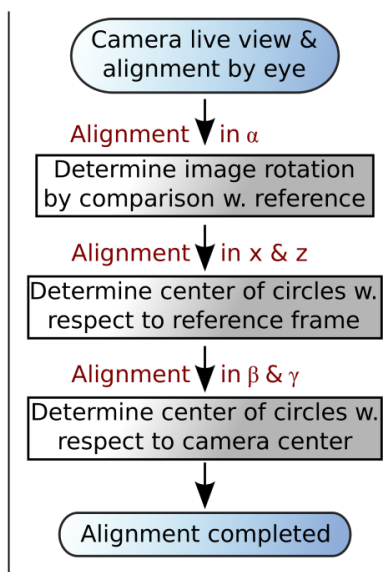

(b)
FIG. 3. Image taken from the MATLAB program during the alignment process and diagram showing the relevant stages throughout the alignment process. (a) Upper panel: Drawing of the region of the manipulator that can be seen with the camera. The alignment along several crucial axes is indicated by the red arrows (rotation around $\gamma$ not shown). Lower panel: Image of the manipulator obtained by the camera during the alignment process. The alignment program has recognised both circles on the manipulator and highlighted them in red. (b) Diagram showing the several stages that the software passes through in order to assist the user during the alignment. Starting with a first coarse alignment by eye using the live view from the camera any misalignment along each axis is dealt with step by step.

completed, the pressure returns to less than $1 \cdot 10^{-10} \mathrm{mbar}$ within a matter of minutes.

Having a live view of the manipulator is helpful in identifying misalignment. However, to make the process quantitative and repeatable, we have developed a script using image processing algorithms. The script was implemented in MATLAB and is particularly useful for inexperienced users. It is programmed to recognise key features of the manipulator, from which the misalignment can be determined using simple geometrical considerations, together with the known dimensions and camera properties. Any misalignment can be corrected by moving the manipulator accordingly. The process is illustrated in Fig. 3(a). We use two concentric circles as the primary alignment aid. Circles are easily identified using standard software and they can be seen in the upper panel of Fig. 3(a). The lower panel of Fig. 3(a) shows an image, with the features recognised by the software and highlighted in red.

The whole procedure involves four stages which are also illustrated in Fig. 3(b):

1. The live-view from the camera is used to align the manipulator by eye. For this purpose a cross-hair is superimposed onto the camera image.

2. Any misalignment in $\alpha$ is determined by the software. For this, a reference image is compared to the camera image and the rotation between these two images is calculated.

3. Alignment of $x$ and $z$ (see Fig. 3(a)): To do this, it is necessary to separate out the effects of $x / z$ and $\beta / \gamma$ misalignments. The position of the two circles with respect to the manipulator reference frame origin (the centre of rotation in $\beta$ and $\gamma$ ) is given by the dimensions of the manipulator. The program uses these dimensions to locate the origin, which is unmoved by changes in $\beta$ and $\gamma$. If it is mis- 
aligned, it must be due to an $x / z$ misalignment, which is then determined and communicated to the user.

4. Now that only a misalignment in $\beta / \gamma$ remains, this misalignment is calculated by the program using elementary geometry and the positions of the centres of the two circles.

At each stage, the program waits for the user to correct any reported misalignments by moving the manipulator accordingly and to agree to proceed. Note that alignment in $y$ is not included in the software, since this parameter is not crucial for the sample transfer. By means of the software, misalignments can be determined quickly and to a greater accuracy so that the alignment process can be automated to a degree. The alignment process using the software typically takes no longer than a couple of minutes.

In summary, we have shown that a commercial webcam can be adapted to provide live views from within a vacuum chamber in order to align a sample transfer system. Image processing software, which uses the pictures taken by the camera, has proven to be a valuable tool in order to automate the alignment process. The alignment software can also be easily adapted for other systems by modifying several parameters such as the dimensions of the manipulator to the ones of a new system.

One of us (A.T.) acknowledges financial support provided by the FWF (Austrian Science Fund) within Project No. J3479N20.

The image processing code developed for the alignment is published under the BSD 3-Clause license and available under http://www.mathworks.com/matlabcentral/fileexchange/ 56442-sample-position-alignment.

${ }^{1}$ D. Farías and K.-H. Rieder, Rep. Prog. Phys. 61, 1575 (1998).

${ }^{2}$ P. Kraus, A. Tamtögl, M. Mayrhofer-Reinhartshuber, G. Benedek, and W. E. Ernst, Phys. Rev. B 87, 245433 (2013).

${ }^{3}$ A. Tamtögl, E. Bahn, J. Zhu, P. Fouquet, J. Ellis, and W. Allison, J. Phys. Chem. C 119, 25983 (2015).

${ }^{4}$ P. R. Kole, "Dynamics and morphology of metal and metal oxide surfaces," Ph.D. thesis, University of Cambridge, 2011.

${ }^{5}$ S. C. Delaquis, R. Gornea, S. Janos, M. Lüthi, C. R. von Rohr, M. Schenk, and J. L. Vuilleumier, J. Instrum. 8, T12001 (2013). 